\title{
Gating Reaction Mechanisms for NMDA Receptor Channels
}

\author{
Yu Zhou and Anthony Auerbach \\ Center for Single Molecule Biophysics and Department of Physiology and Biophysics, State University of New York, Buffalo, New York 14214
}

\begin{abstract}
NMDA receptors (NMDARs) mediate the slow component of excitatory transmission in the CNS and play key roles in synaptic plasticity and excitotoxicity. We investigated the gating reaction mechanism of fully liganded NR1/NR2A recombinant NMDARs (expressed in Xenopus oocytes) by fitting all possible three-closed/two-open-state, noncyclic kinetic schemes to currents elicited by saturating concentrations of glutamate plus glycine. The adequacy of each scheme was assessed by maximum likelihood values and autocorrelation coefficients of single-channel currents, as well as by the predicted time courses of transient macroscopic currents. Two schemes provided the best description for NMDAR gating at both the single-channel and macroscopic levels. These two schemes had coupled open states, only one gateway between the closed and open aggregates, and at least two preopening closed states. These two models could be condensed into a cyclic reaction mechanism. Using a linear reaction scheme, the overall "gating" rates (from the initial stable closed state to the final stable open state) are 177 and $4.4 \mathrm{~s}^{-1}$.
\end{abstract}

Key words: channel; kinetics; oocyte; glutamate receptor; glutamate; NMDA receptor

\section{Introduction}

NMDA receptors (NMDARs) are glutamate-gated ion channels that are $\mathrm{Ca}^{2+}$ permeable and experience voltage-dependent block by $\mathrm{Mg}^{2+}$ (Mayer et al., 1984; Nowak et al., 1984; Wollmuth and Sakmann, 1998; Wollmuth et al., 1998). These special properties, and their slow activation and deactivation rates, make NMDARs an important contributor to long-term synaptic plasticity and excitotoxicity (Hollmann and Heinemann, 1994; Dingledine et al., 1999). NMDARs are typically composed of two NR1 and two NR2 subunits that bind glycine and glutamate, respectively (Benveniste and Mayer, 1991a; Clements et al., 1992; Behe et al., 1995; Rosenmund et al., 1998). Structural studies indicate that the binding of agonists causes a conformational change in the S1-S2 binding site domains of the protein (Sun et al., 2002; Furukawa and Gouaux, 2003), which is likely to be an early conformational event that leads to the isomerization of the ion channel domain from an ion-impermeable ["closed" (C)] conformation to an ion-permeable ["open" $(\mathrm{O})$ ] conformation. However, the details of the molecular events that constitute the global conformational change in the protein ("gating") remain unknown. Kinetic models are useful in this regard, because they serve to encode the energy landscape for dynamic changes in protein structure. Furthermore, such models have predicative value and can be used to both interpret and forecast the behavior of NMDARs at synapses (Popescu and Auerbach, 2004; Popescu et al., 2004).

Kinetic models of NMDAR activity that have been derived by fitting macroscopic currents have focused mainly on agonist-

Received April 14, 2005; revised July 5, 2005; accepted July 5, 2005.

This work was supported by National Institutes of Health Grant 5R01NS036554-07 to A.A. We thank M. Teeling for technical assistance and P. Seeburg and T. Kuner for the NMDA receptor subunit CDNAs.

Correspondence should be addressed to Anthony Auerbach, Department of Physiology and Biophysics, State University of New York, 324 Cary Hall, Buffalo, NY 14214. E-mail: auerbach@buffalo.edu.

D0I:10.1523/JNEUROSCI.1471-05.2005

Copyright $\odot 2005$ Society for Neuroscience $\quad$ 0270-6474/05/257914-10\$15.00/0 binding steps (Benveniste and Mayer, 1991a; Clements et al., 1992; Lester and Jahr, 1992). In these schemes, the gating conformational change is usually represented simply as a single-step reaction. Although these models can explain the time course of macroscopic NMDAR currents, they fail to describe the complex kinetics of single-channel NMDAR currents (Gibb and Colquhoun, 1991, 1992; Stern et al., 1992). Recently, more complex models for fully liganded NMDAR gating have been proposed (Banke and Traynelis, 2003; Popescu and Auerbach, 2003). In the Banke and Traynelis (BT) model (Banke and Traynelis, 2003), fully liganded (NR1/NR2B) NMDARs can occupy four closed and only one open state. Based on the observation that partial agonists of each of the two classes of binding sites only change one component of the closed-time distribution, Banke and Traynelis proposed a gating mechanism in which the NR1 and NR2 subunit pairs isomerize independently, followed by an additional conformational change that changes the conductance of the pore. In the model of Popescu and Auerbach (2003), fully liganded (NR1/NR2A) NMDARs can adopt multiple activation modes, each of which has three closed and two coupled open states. Although these two models are similar, it remains important to define more precisely a kinetic scheme for NMDAR gating that can be related to specific structural changes in the protein, as well as be used to make predictions about the time course and amplitudes of synaptic currents (Popescu et al., 2004).

Here, we present a statistical analysis of single-channel and macroscopic currents induced by saturating agonists from NR1a/ NR2A NMDARs expressed in Xenopus oocytes. All possible three-closed/two-open (3C2O) noncyclic schemes were fitted to quantitatively assess their abilities to describe fully liganded NMDAR gating. The two optimal schemes have coupled open states, the first of which is preceded by at least two closed states, and only one entry/exit state between the closed and open aggregates. These schemes can be combined into a cyclic model in which a closed, fully liganded NMDAR can choose between two 
preopening conformational pathways before reaching an ionconducting state.

\section{Materials and Methods}

\section{Expression of cRNA in oocytes}

RNA was transcribed in vitro from cDNA by standard methods. To express NMDARs, the RNAs of rat NR1a and NR2A were coinjected at a nominal ratio of 1:1 in Xenopus oocytes. Patch-clamp recordings were made 3-10 d after injection. A detailed description of the molecular biology and expression protocols has been described previously (Premkumar and Auerbach, 1996).

\section{Electrophysiological recording}

Single-channel and macroscopic currents were recorded from outsideout or cell-attached patches (Hamill et al., 1981). The temperature was $23-25^{\circ} \mathrm{C}$. Agonists were contained in the pipette solution (cell-attached patches) or applied to outside-out patches by using a fast perfusion system. The perfusion pipette was pulled from double-barreled glass tubing (tip diameter, $200 \mu \mathrm{M}$ ) and attached to a bimorph actuator (model QP22B; ACX, Cambridge, MA) using epoxy resin. External solutions were delivered to each of the two barrels by using air pressure. The actuator was controlled by QUB software (http://www.qub.buffalo.edu) to move the solution interface across the tip of patch pipette. The command voltage pulses were low-pass filtered at $150 \mathrm{~Hz}$. The actual solution-exchanging rate was routinely measured on the same patch pipette at the end of each recording by open-tip response (Jonas, 1995). The $10-90 \%$ rise time of this system was $\sim 200 \mu$ s (see Fig. $4 a$, inset).

Patch pipettes were pulled from borosilicate capillary glass (Sutter Instruments, Novato, CA), coated with Sylgard 184 (Dow Corning, Midland, MI), and fire-polished before recording. The pipette resistance ranged from 0.5 to $2 \mathrm{M} \Omega$ for macropatches and 10 to $20 \Omega$ for singlechannel recording. The pipette solution contained the following (in $\mathrm{mM}$ ): $100 \mathrm{Na}$ gluconate, $10 \mathrm{NaCl}, 10$ BAPTA, 10 HEPES, $2 \mathrm{~K}_{2} \mathrm{ATP}$, and 0.25 GTP. The $\mathrm{pH}$ of the pipette solution was adjusted to 7.3 by using $\mathrm{NaOH}$. The extracellular solution contained the following (in $\mathrm{mm}$ ): $100 \mathrm{NaCl}, 2$ $\mathrm{KCl}, 1$ EDTA (to eliminate $\mathrm{Mg}^{2+}$ block), $10 \mathrm{~N}$-(2-hydroxyethyl)piperazine- $N[\mathrm{E}$-(4-butanesulfonic acid)], $\mathrm{pH}$ adjusted to 8 (to minimize proton inhibition) by using $\mathrm{NaOH}$. All chemicals were purchased from Sigma (St. Louis, MO) or Aldrich (Milwaukee, WI). Currents were amplified (PC-505B; Warner Instruments, Hamden, CT), low-pass filtered at $20 \mathrm{kHz}$ (eight-pole Bessel; LPF-8; Warner Instruments), and digitized at $40 \mathrm{kHz}$ directly to a hard disk using a MIO-16E digital board (National Instruments, Austin, TX).

\section{Data analysis}

Conductance level detection and amplitude histograms. The conductance levels of NMDARs were detected by variance-mean analysis (Patlak, 1988). A window of length $N$ sampling points was centered over each sample of current, and the variance $\left(\sigma^{2}\right)$ and mean amplitude $(I)$ of the current within the window were calculated. The window was advanced by one sample, and the pair of $\sigma^{2}$ and $I$ values was again calculated, with this process repeated for the entire record. If the sliding window covers more than one conductance level, the variance will increase. Changes in conductance were identified by the transitions in the variance points relative to that of the baseline $\left(\sigma_{\mathrm{b}}{ }^{2}\right)$. This analysis was performed by a program written in OriginC language (OriginLab, Northampton, MA).

Simulation, idealization, and maximum likelihood fitting. QUB software was used for simulation, idealization, and modeling of singlechannel currents. Data were corrected for slow and nonperiodic baseline drifts using manually specified piecewise linear functions and then idealized by a segmental $k$-means method (Qin, 2004). Clusters of singlechannel current openings were chosen by excluding the longest closed events (desensitization) by invoking a critical shut time $\left(t_{\text {crit }}\right)$ that was based on an equal number of misclassified events (Magleby and Pallotta, 1983; Clapham and Neher, 1984). Bursts from patches having only one active channel were concatenated as a continuous "active-time" record. To test for kinetic homogeneity, this record was divided into $1 \mathrm{~s} \mathrm{segments}$ and idealized by using a two-state $(\mathrm{C} \rightleftarrows \mathrm{O})$ model to calculate a segment mean open and closed time. The kinetic modeling was based on clusters rather than the segmented record. The currents were idealized using the segmental k-means (SKM) algorithm and the rate constants for a given model were estimated by using a full maximum likelihood approach (Qin et al., 1996; Qin, 2004) after imposing a "dead time" of two to three sampling intervals $(50-75 \mu \mathrm{s})$. The dwell times given by the SKM method are integer multiples of the sampling interval $(25 \mu \mathrm{s})$.

Correlation analysis. To help ascertain the connectivity of the states in the model, two-dimensional (2D) dependency plot analysis (Magleby and Song, 1992) and open-open, closed-closed, and open-closed autocorrelation analyses (Fredkin et al., 1985; Labarca et al., 1985) were performed on idealized single-channel data without correcting for missed events. The autocorrelation coefficient for two intervals $T_{1}$ and $T_{2}$ in an idealized single-channel current is as follows:

$$
\begin{aligned}
\rho\left(T_{1}, T_{2}\right) & =\frac{\operatorname{cov}\left(T_{1}, T_{2}\right)}{\sqrt{\operatorname{var}\left(T_{1}\right) \operatorname{var}\left(T_{2}\right)}} \\
& =\frac{E\left(T_{1}, T_{2}\right)-\mu_{1} \mu_{2}}{\sqrt{\left[E\left(T_{1}^{2}\right)-\mu_{1}^{2}\right]\left[E\left(T_{2}^{2}\right)-\mu_{2}^{2}\right]}},
\end{aligned}
$$

where the means are $\mu_{1}=E\left(T_{1}\right)$ and $\mu_{2}=E\left(T_{2}\right)$.

Thus, the autocorrelation coefficient between an open (or closed) interval $T$ and the $k$ th open (or closed) interval $T^{\prime}$ behind is as follows:

$$
\rho(k)=\frac{\left[E\left(T T^{\prime}\right)-\mu^{2}\right]}{\sigma^{2}},
$$

where $\mu$ is the mean and $\sigma^{2}$ is the variance of open (or closed) dwell times in the recording. The autocorrelation coefficient between an open interval $T_{\mathrm{O}}$ and the $k$ th closed interval $T_{\mathrm{C}}$ behind is as follows:

$$
\rho(k)=\frac{\left[E\left(T_{\mathrm{O}} T_{\mathrm{C}}\right)-\mu_{\mathrm{open}} \mu_{\text {closed }}\right]}{\sqrt{\sigma_{\mathrm{open}}^{2} \sigma_{\text {closed }}^{2}}} .
$$

According to theorem 5.1 of Fredkin et al. (1985), the autocorrelation coefficient decays geometrically with increasing lag $k$ if there is more than one entry/exit state between open and closed aggregates. The correlation coefficients were used to test the activation mechanisms of ACh receptor in adult frog muscle (Colquhoun and Sakmann, 1985).

The $2 \mathrm{D}$ dependency, $d\left(t_{\mathrm{O}}, t_{\mathrm{S}}\right)$, is as follows:

$$
d\left(t_{\mathrm{O}}, t_{\mathrm{S}}\right)=\frac{f\left(t_{\mathrm{O}}, t_{\mathrm{S}}\right)-f^{\prime}\left(t_{\mathrm{O}}, t_{\mathrm{S}}\right)}{f^{\prime}\left(t_{\mathrm{O}}, t_{\mathrm{S}}\right)},
$$

where $f\left(t_{\mathrm{O}}, t_{\mathrm{S}}\right)$ is the observed $2 \mathrm{D}$ probability density function and $f$ $'\left(t_{\mathrm{O}}, t_{\mathrm{S}}\right)=f_{\mathrm{O}}\left(t_{\mathrm{O}}\right) f_{\mathrm{S}}\left(t_{\mathrm{S}}\right)$ is the expected $2 \mathrm{D}$ probability density function if there is no dependence between paired open and closed intervals. To create the 2D dwell-time distribution of adjacent open and shut intervals, the logs of the durations of each open interval and the following closed interval were used to locate a bin on the $x-y$ plane, with the $z$-axis indicating the square root of the number of interval pairs in each bin (Sigworth and Sine, 1987). The 2D dwell-time distributions were then smoothed by using the weighted average value of nine bins in a $3 \times 3$ array with three bins per side moving through the $x-y$ plane (Song and Magleby, 1994). The weighted average is calculated as follows:

$\bar{a}=\frac{0.707\left(a_{11}+a_{13}+a_{31}+a_{33}\right)+\left(a_{12}+a_{21}+a_{22}+a_{23}+a_{32}\right)}{7.828}$,

where $a_{11}$ through $a_{33}$ are the elements in the moving array:

$$
\begin{array}{lll}
a_{11} & a_{12} & a_{13} \\
a_{21} & a_{22} & a_{23} \\
a_{31} & a_{32} & a_{33}
\end{array} .
$$

Programs written in OriginC language were used for the correlation analyses and 2D dependency plots. Autocorrelation coefficients of openopen, closed-closed, and open-closed interval pairs predicted by kinetic models (Colquhoun and Hawkes, 1987) were calculated by using a worksheet written in Maple 7 (Waterloo Maple, Waterloo, Ontario, Canada). 
Macroscopic current analysis. Normalized macroscopic currents were computed from each kinetic model (Colquhoun and Hawkes, 1995a,b) by using a Maple 7 worksheet. The 5-95\% rising and decay phases of the computed and experimentally recorded macroscopic currents were compared by eye and by calculating the deviation of simulated response from experimental current by using the following function:

$$
d=\sum \frac{(\text { recorded }- \text { simulated })^{2}}{\mid \text { simulated } \mid} .
$$

All data are expressed as the mean \pm SD.

\section{Results}

Single-channel activity of NMDARs

Under physiological conditions, the kinetic behavior of NMDARs at the singlechannel level is difficult to interpret because of the binding of multiple agonists and inhibition by several types of ions, including protons and $\mathrm{Mg}^{2+}$. To simplify matters, we used supermaximal concentrations of glutamate and glycine (to saturate the binding sites at steady state or to maximally accelerate binding after a concentration jump) and high $\mathrm{pH}$, divalent cation-free extracellular solutions (to reduce or eliminate ion inhibition). Figure $1 a$ shows single-channel currents from an outside-out patch exposed to $1 \mathrm{~mm}$ glutamate and $100 \mu \mathrm{M}$ glycine. Figure $1 b$ shows the corresponding variance-mean amplitude histogram fitted by the sum of two Gaussian components, one for the ion-conductive level and the other for the nonconductive level. Although brief superconductance- and subconductance- a.

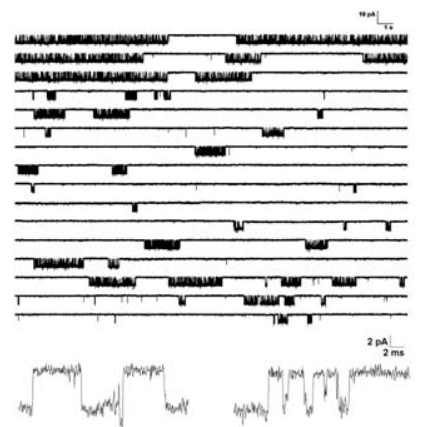

c.

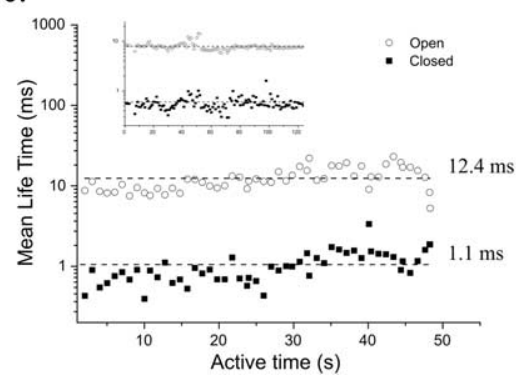

b.

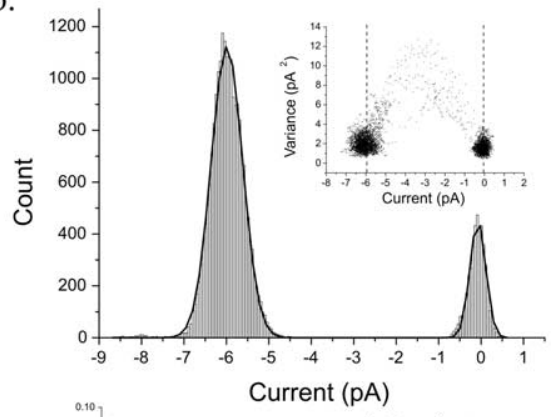

d.

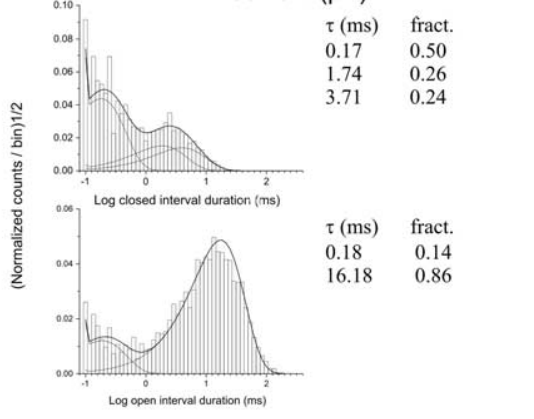

Figure 1. Single-channel activity of NMDARs elicited by $1 \mathrm{~mm}$ glutamate and $100 \mu \mathrm{m}$ glycine in the presence of $1 \mathrm{~mm}$ EDTA. $\boldsymbol{a}$, Continuous record from an outside-out patch at $-80 \mathrm{mV}$. Occasional alternative conductance levels are displayed on an expanded time scale below. $\boldsymbol{b}$, Amplitude histogram constructed from activation bursts and the corresponding mean-variance analysis (inset, 10-sample sliding window). There is primarily one conducting amplitude level with a mean of $5.99 \mathrm{pA}$. c, The mean closed and open durations for each second of active time are stable. An interval duration stability analysis from a cell-attached patch is shown (inset). $\boldsymbol{d}$, Duration distributions of intracluster closed (top) and open (bottom) intervals. Mean lifetimes and relative areas of each exponential component are shown (right). fract., Fraction.

Table 1. Time constants and fractional amplitudes of closed and open intervals within clusters

\begin{tabular}{llllll}
\hline Patch No. & $\tau_{\mathrm{c} 1}[\mathrm{~ms}(\%)]$ & $\tau_{\mathrm{c} 2}[\mathrm{~ms}(\%)]$ & $\tau_{\mathrm{c3}}[\mathrm{ms}(\%)]$ & $\tau_{\mathrm{o} 1}[\mathrm{~ms}(\%)]$ & $\tau_{\mathrm{o2}}[\mathrm{ms}(\%)]$ \\
\hline 12170303 & $0.17(50)$ & $1.74(26)$ & $3.71(24)$ & $0.18(14)$ & $16.18(86)$ \\
12170304 & $0.14(54)$ & $0.99(12)$ & $3.16(34)$ & $0.16(11)$ & $14.10(89)$ \\
12170305 & $0.17(50)$ & $1.48(18)$ & $3.85(32)$ & $0.18(12)$ & $11.54(88)$ \\
12170306 & $0.16(53)$ & $1.45(12)$ & $3.41(35)$ & $0.19(9)$ & $12.62(91)$ \\
\hline
\end{tabular}

Distributions of intracluster closed and open intervals recorded from outside-out patches were best fitted by three and two exponential components, respectively (Fig. $1 d$, Table 1 ). The three closed components had mean time constants (relative areas) of $0.16 \pm 0.014 \mathrm{~ms}(52 \pm 2.1 \%), 1.42 \pm 0.31 \mathrm{~ms}(17 \pm 6.6 \%)$, and $3.53 \pm 0.31 \mathrm{~ms}(31 \pm 5.0 \%)$. The corresponding values for the two open components were $0.18 \pm 0.012 \mathrm{~ms}(12 \pm 2.1 \%)$ and $13.61 \pm 2.01 \mathrm{~ms}(88 \pm 2.1 \%)(n=4)$. These distributions are similar to those of M-mode NR1a/NR2A NMDARs expressed in HEK cells (Popescu and Auerbach, 2003). However, the mean time constants of the two briefest closed components were longer than those recorded in the presence of low concentrations of agonist and divalent cations (Stern et al., 1994; Wyllie et al., 1998). It is possible that this difference is attributable to the absence of fast $\mathrm{Mg}^{2+}$ block in our divalent cation-free solution.

The interval duration distributions of intracluster, singlechannel events recorded from cell-attached patches were also well fitted by three closed and two open components. The mean time constants (relative areas) of three closed components were $0.075 \pm 0.021 \mathrm{~ms}(50 \pm 1 \%), 0.48 \pm 0.1 \mathrm{~ms}(28 \pm 7 \%)$, and $4.29 \pm 0.14 \mathrm{~ms}(22 \pm 7 \%)$. For the two open components, these values were $0.42 \pm 0.23 \mathrm{~ms}(14 \pm 9 \%)$ and $10.63 \pm 4.41 \mathrm{~ms}(86 \pm$ $12 \%)(n=3)$. The distributions from cell-attached and outsideout recordings were similar. The kinetic studies described below tained from data recorded from cell-attached patches (Fig. 1c, inset). 
were mainly derived from analyses of currents from outside-out patches.

\section{Kinetic modeling of single-channel data}

The duration distributions of intraburst events indicate that a parsimonious activation scheme for NMDARs must have three closed and two open states (Colquhoun and Hawkes, 1981, 1982). These five states can be arranged to constitute 15 noncyclic kinetic models, six of which have coupled open states (Table 2). All 15 models were fitted to the intracluster interval durations (four patches) by using a full maximum likelihood approach, with all eight rate constants allowed to vary. Seven of these models gave an equivalent maximum log likelihood (LL) value that was higher ( $>10$ LL units) than those from the other eight models (Table 3). The models having coupled open states always gave

Table 2. Noncyclic models having three closed and two open states

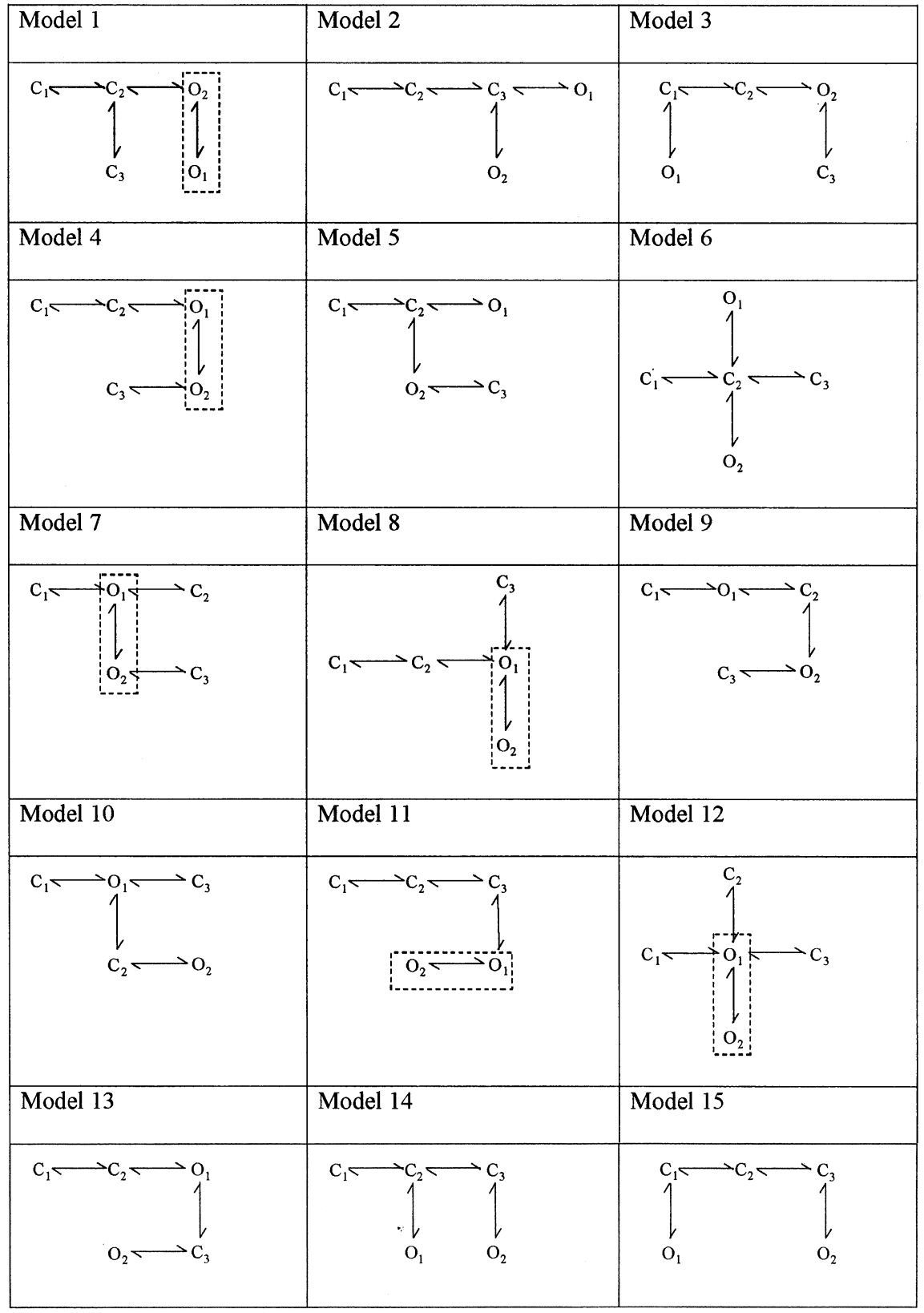

higher log likelihood values than those with uncoupled open states.

The seven best models can be further classified by their connectivities. In models 4,5 , and 7 , there are two entry/exit states between the open and closed aggregates $\left(\mathrm{O}_{1}\right.$ and $\left.\mathrm{O}_{2}\right)$, whereas in models $1,8,11$, and 12 , there is only one $\left(\mathrm{O}_{1}\right)$. A model with connectivity $>1$ predicts the existence of an autocorrelation between open, closed, or open-closed interval pairs in singlechannel data (Fredkin et al., 1985; Colquhoun and Hawkes, 1987).

Autocorrelation coefficients predicted by models 4, 5, and 7 were calculated by a method provided by Colquhoun and Hawkes (1987). As shown in Figure $2 a$, model 4 predicts a negative correlation between open-closed pairs, a positive (but smaller) correlation between open-open pairs and a weak, positive correlation between closed-closed pairs. Similar predictions hold for the other two models having a connectivity of 2 (models 5 and 7). The open-closed correlation coefficients for lag $k=1$ predicted by models 4,5 , and 7 were approximately -0.03 , which could be readily detected in data simulated from these models when the number of intervals was $>9000$ (Fig. 2b). However, there were no significant autocorrelations between open-open, closed-closed, or openclosed interval pairs in the experimental NMDAR currents. As illustrated in Figure $2 d$, all three types of correlation coefficients in an activation cluster recorded from an outside-out patch were normally distributed around zero. Similar results were obtained from several long activation clusters having $>9000$ intervals, from cell-attached patches (Fig. $2 d$, inset). Moreover, the 2D dependency plots of the experimental current intervals did not show any significant excess or deficit in open-closed pairs within clusters (Fig. $2 c$ ). The above correlation analyses and dependency plots were not corrected for missed events. However, the consequence of this omission may not be crucial, because no correlations were detected in the experimental data. Thus, the results indicate that the activation model for NMDAR should have only one entry/exit state through which the open and closed aggregates communicate. Based on these analyses, four models $(1,8,11$, and 12) were selected for additional consideration.

In theory, the full maximum likelihood analysis should take into account all of the information in a single-channel record, including dependency between successive events in the record (Colquhoun and Hawkes, 1995a). However, spurious events generated by noise, errors in detection, and rare channel behaviors limit the usefulness of the LL discriminator. Dependency and autocorrelation analyses are valuable complements to maximum like- 
Table 3. Optimal parameters $\left(\mathrm{s}^{-1}\right)$ for models having the highest maximum log likelihood values

\begin{tabular}{|c|c|c|c|c|}
\hline Patch No. & 12170303 & 12170304 & 12170305 & 12170306 \\
\hline Model 1 & 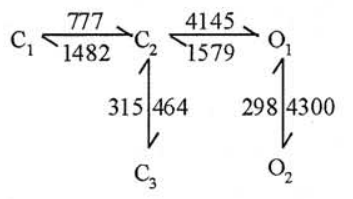 & 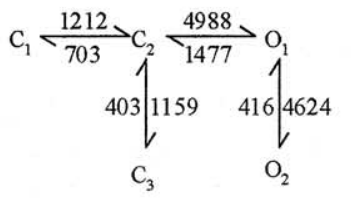 & 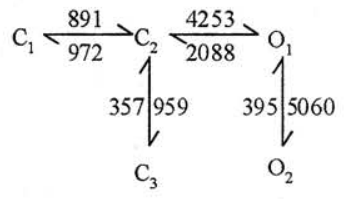 & 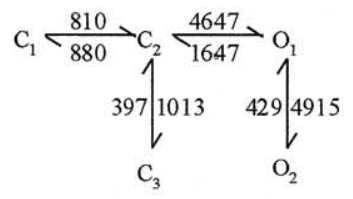 \\
\hline Model 4 & 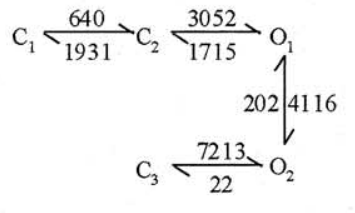 & 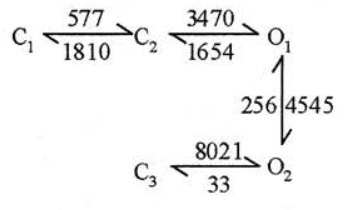 & 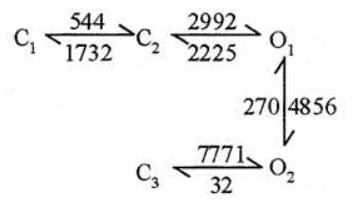 & 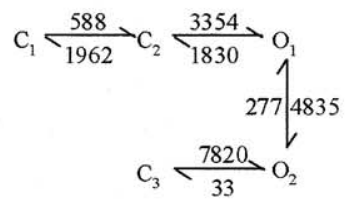 \\
\hline Model 5 & 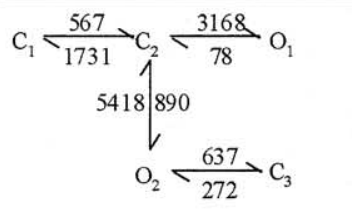 & $\begin{aligned} \mathrm{C}_{1} \underset{1569}{\stackrel{486}{\longrightarrow}} \mathrm{C}_{2} \underset{5513}{\stackrel{39636}{862}} \mathrm{O}_{1} \\
\mathrm{O}_{2} \underset{542}{\stackrel{857}{\longrightarrow} \mathrm{C}_{3}}\end{aligned}$ & 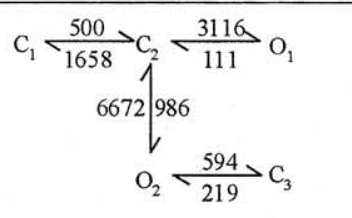 & 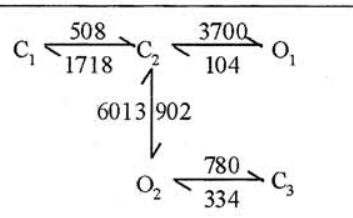 \\
\hline Model 7 & 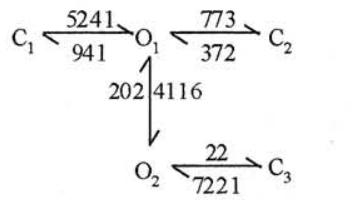 & 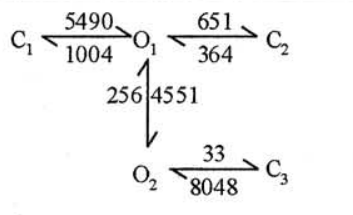 & 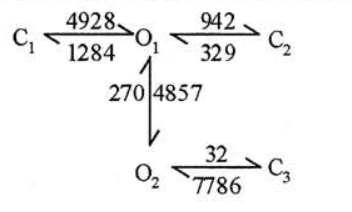 & 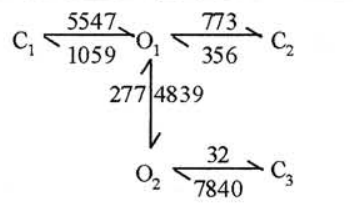 \\
\hline Model 8 & 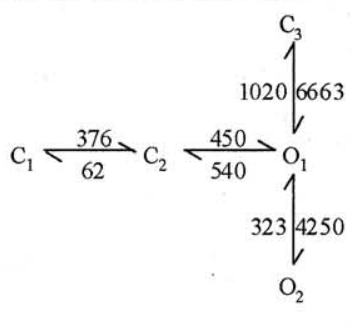 & 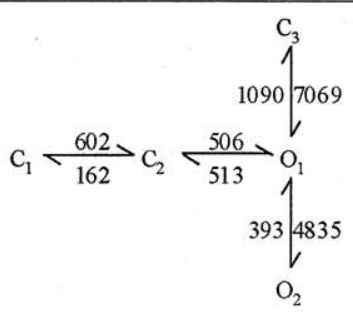 & 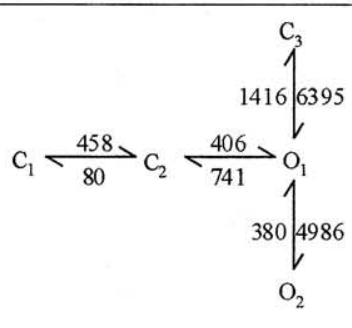 & 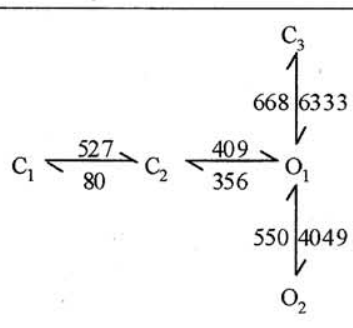 \\
\hline Model 11 & 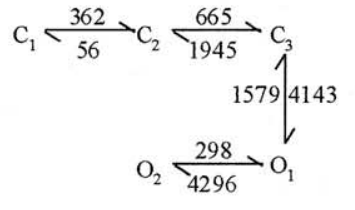 & $\begin{aligned} \mathrm{C}_{1} \underset{159}{\stackrel{613}{\longrightarrow}} \mathrm{C}_{2} \underset{1830}{\stackrel{672}{\underset{1874}{\longrightarrow}} \mathrm{C}_{3}} & \stackrel{1}{4959} \\
& \mathrm{O}_{2} \underset{4624}{\stackrel{416}{\longrightarrow}} \mathrm{O}_{1}\end{aligned}$ & 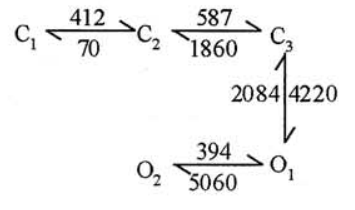 & 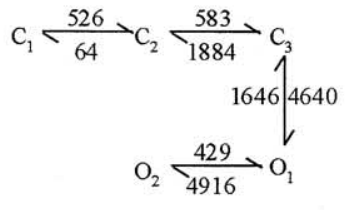 \\
\hline Model 12 & 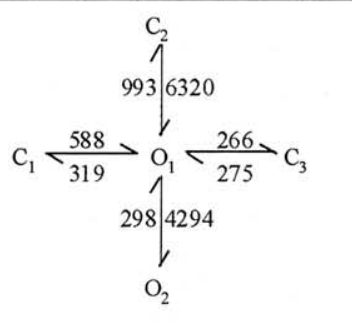 & 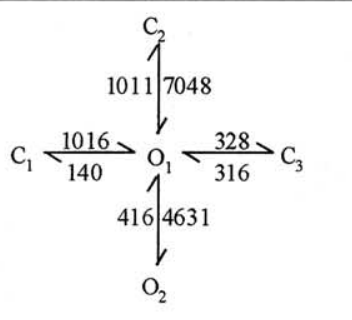 & 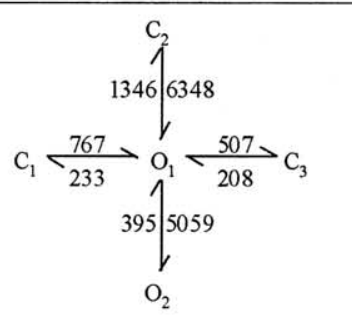 & 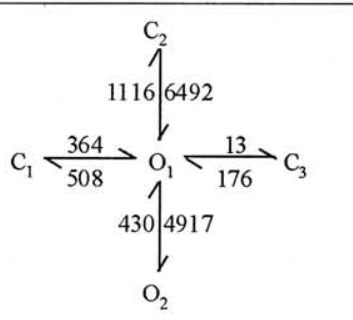 \\
\hline
\end{tabular}

lihood fitting in model selection (Magleby and Song, 1992; Colquhoun and Hawkes, 1995a; Gil et al., 2001).

Predictions of macroscopic current properties

The four best schemes can be further classified by the number of closed states that must be visited (after full binding) before the first open state is reached. This property of the reaction scheme predicts distinguishable time courses of the macroscopic current after the fast application of a high concentration of agonists. As shown in Figure 3, if NMDARs must pass through two closed states (e.g., model 8, starting from $\mathrm{C}_{1}$ ), the rising phase of macroscopic current should be sigmoidal, whereas if receptors have 


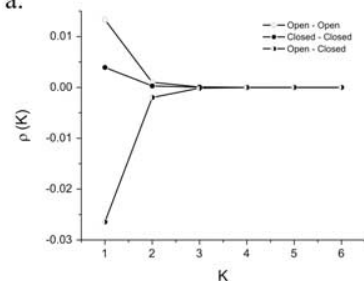

b.

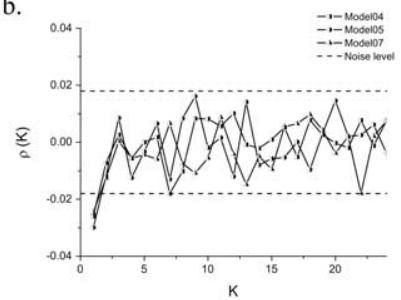

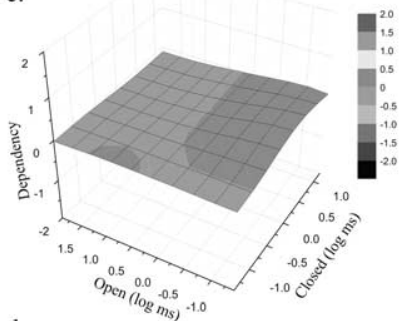

d.

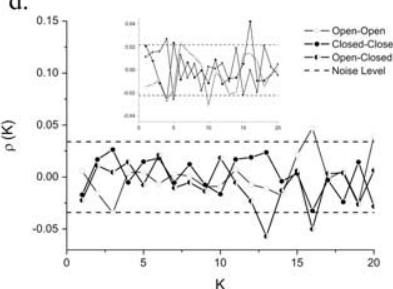

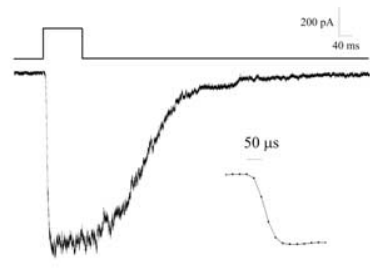

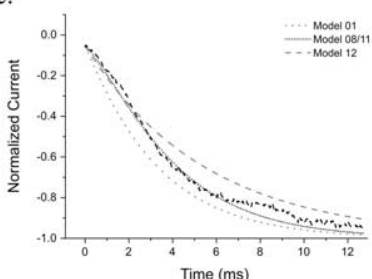

b.

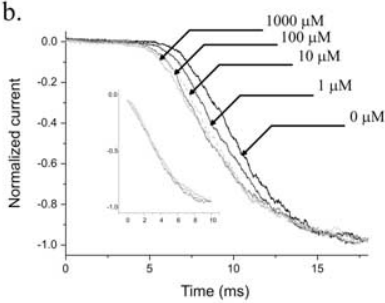

d.

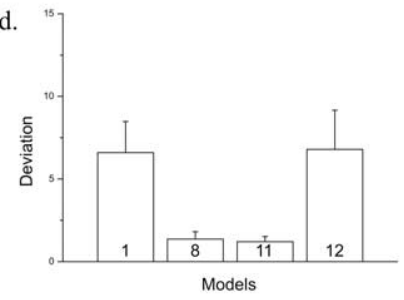

Figure 2. Correlation analyses of NMDAR-idealized single-channel currents, from experimental and simulated currents. $\boldsymbol{a}$, Autocorrelation coefficients of open, closed, and openclosed interval pairs predicted by model 4. $\boldsymbol{b}$, Autocorrelation coefficients of open-closed interval pairs simulated by using models 4,5 , and 7 (total number of events, $n=11,000$ ). The dashed horizontal lines are $\pm 2 / \sqrt{n}$, which is plus or minus twice the SE of the estimate in the case of white noise. c, 2D dependency plot of 7629 intraburst intervals from one outside-out patch. There is no significant dependency between adjacent open and closed intervals. d, Autocorrelation coefficients for the open, closed, and open-closed interval pairs from experimental NMDAR current cluster recorded from an outside-out patch $(n=3274)$. Autocorrelation coefficients for the open- open, closed-closed, and open-closed interval pairs from one activation cluster recorded from a cell-attached patch are shown $(n=9760)$ (inset). K, Step size; $\rho(\mathrm{K})$, autocorrelation coefficient.

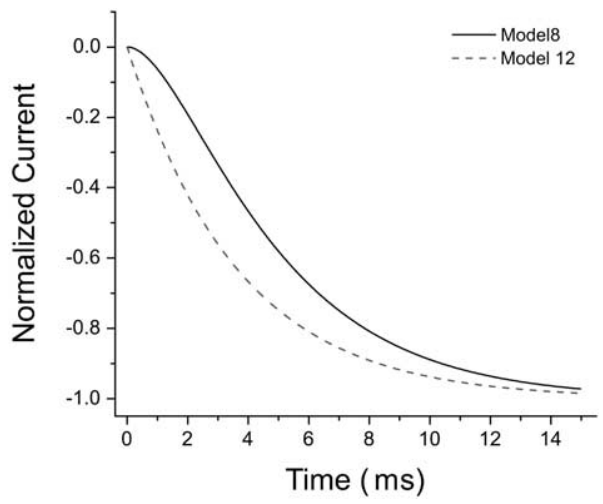

Figure 3. Time course of the macroscopic current predicted by models having different numbers of preopening closed states. The solid line was simulated from model 8 with the assumption that activation starts from $C_{1}$. The dashed line was simulated from model 12 with $C_{3}$ as the starting point. The rate constants used for simulation were obtained by globally fitting activation bursts from four patches.

to pass through only one closed state (e.g., model 12), then the rising phase of macroscopic current should be exponential. We sought to discriminate between models by comparing their predictions on the time course of macroscopic currents induced by a step change in the agonist concentration. Note that the models tested here pertain to only the gating reaction; thus, the NMDAR macroscopic currents should exclude ligand binding and desensitization steps.

Saturating concentrations of agonists were applied by a fast perfusion system (solution change $<200 \mu$ s) on outside-out macropatches (Fig. 4a). The rising phase of evoked macroscopic current was $<15 \mathrm{~ms}$ (Fig. 4 ), which is much faster than the time

Figure 4. The rising phase of NMDAR macroscopic current evoked by fast application of saturating concentrations of agonists is satisfied by models 8 and 11. $\boldsymbol{a}$, Macroscopic current induced by $1 \mathrm{~mm}$ glutamate plus $100 \mu \mathrm{m}$ glycine applied to an outside-out macropatch ( -60 $\mathrm{mV})$. The top trace is the command pulse indicating agonist application. The inset is the opentip response recorded on the same patch pipette, on expanded scale. $\boldsymbol{b}$, Macroscopic currents after a concentration jump from solutions containing different concentrations of glycine with no glutamate to a solution containing $100 \mu \mathrm{m}$ glycine and $1 \mathrm{~mm}$ glutamate. Alignment of the $5-$ $95 \%$ rising phases of the currents is shown (inset). c, Comparison of experimental and simulated macroscopic currents. Only the best result from each model was used for comparison. The simulated currents from models 8 and 11 overlap. $\boldsymbol{d}$, Deviations calculated by Equation 7 of predicted currents from experimental current. Models 8 and 11 provide equivalent and superior descriptions of the experimental current. Error bars indicate SE.

constant of NMDAR desensitization (>100 ms) (Vicini et al., 1998; Villarroel et al., 1998). To confirm that the rising phase was independent of binding steps, a series of jumps from solutions containing various concentrations of glycine to a solution with 1 mM glutamate plus $100 \mu \mathrm{M}$ glycine was performed (Fig. $4 b$ ). The rising phases were identical, although the glycine binding steps were different in these jumps. This indicates that that glycine binding did not contribute significantly to the rising phase of NMDAR current at saturating concentration. We could not perform the complementary experiment with glutamate binding, because it is difficult to eliminate background contamination of glycine (Kleckner and Dingledine, 1988). However, the glutamate equilibrium dissociation constant is $\sim 3 \mu \mathrm{M}$, and the association rate constant is $\sim 10^{8} \mathrm{M}^{-1} \cdot \mathrm{s}^{-1}$ (Benveniste and Mayer, 1991a,b; Lester and Jahr, 1992; Popescu et al., 2004), which suggests that we could expect full saturation of the binding sites within $\sim 200 \mu \mathrm{s}$. We therefore conclude that the rising phase of the macroscopic current evoked by the fast application of saturating concentrations of agonists can be used to examine the predictions by various models of the gating reaction.

The best predictions by models $1,8,11$, and 12 are superimposed on normalized NMDAR macroscopic currents in Figure $4 c$. The rising phase of NMDAR current was clearly sigmoidal, in agreement with models 8 and 11 using the rate constants estimated from modeling single-channel interval data. Models 1 and 12 both predicted an exponential rising phase, although in model 12, there were two closed states before the first open state. Overall, the deviations of the predictions by models 8 and 11 were much lower than those of predictions by models 1 and 12 (Fig. 4d).

\section{Combining the noncyclic models}

After the above three rounds of model selection, we were left with two noncyclic, 3C2O models that provided adequate and equally 
a.

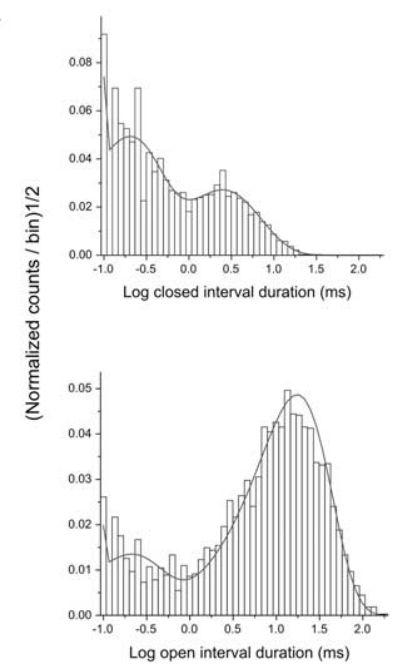

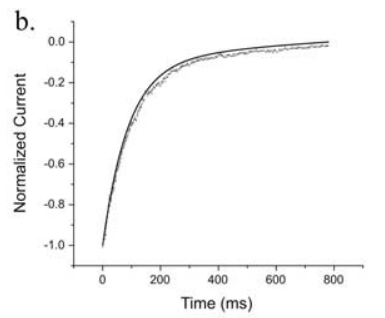

c.

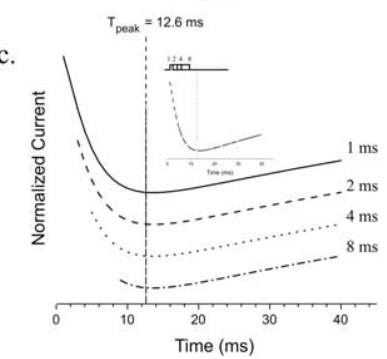

Figure 5. Both microscopic and macroscopic currents are described by model 16. $\boldsymbol{a}$, Histograms of intraburst interval distributions. The solid lines are calculated from the model. $\boldsymbol{b}$, Simulated (solid line) and experimental current decays after a $100 \mathrm{~ms}$ pulse of $1 \mathrm{~mm}$ glutamate plus $100 \mu \mathrm{m}$ glycine. Binding and desensitization states (both connected to $C_{1}$ ) were added to model 16. The dashed line is the fit of experimental current by the sum of two exponentials. $c$ Simulations of the current response to short pulses $(1,2,4$, and $8 \mathrm{~ms})$ of saturating agonists (model 16). The time to peak ( $\left.T_{\text {peak }}\right)$ is the same for all pulse durations. Currents are staggered vertically for display purposes. The inset is the superimposed view of these traces.

good descriptions for the activation of NMDAR, at both the microscopic and macroscopic levels. These two models differ in the disposition of closed state $\mathrm{C}_{3}$, which is either connected only to $\mathrm{O}_{1}$ (model 8) or interposed between $\mathrm{C}_{2}$ and $\mathrm{O}_{1}$ (model 11). We could not use statistical tests to further distinguish these two models; hence, we combined them into a single cyclic scheme that preserves the key features of both two noncyclic models, namely coupled open states, a single gateway between closed and open aggregates, and multiple preopening closed states.

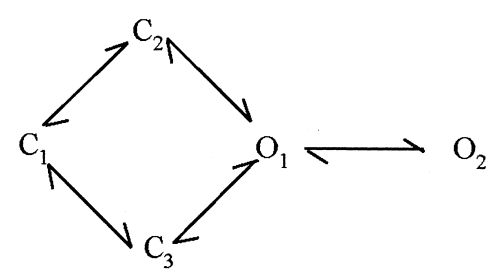

Model 16

Detailed balance was enforced for the cycle in model 16, which had one extra free parameter compared with models 8 and 11 .

Figure $5 a$ shows that the experimental intracluster closed (top panel) and open (bottom panel) distributions are in good agreement with those predicted by model 16 (continuous lines). In addition, the autocorrelation function of data simulated from this model has the same flat shape as those obtained from NMDAR activation clusters. Model 16 also accurately predicts the time course of macroscopic currents evoked by fast application of agonists when $\mathrm{C}_{1}$ is the starting state (data not shown). Although neither the LL values obtained from single-channel kinetic modeling nor the deviations of the macroscopic currents from those predicted by the model were significantly improved by the extra free parameter, model 16 is appealing in that it provides an unambiguous gating reaction mechanism for NMDARs.

According to this scheme, there are two alternative conformational pathways in the gating reaction $\left(\mathrm{C}_{1} \mathrm{C}_{2} \mathrm{O}_{1}\right.$ or $\left.\mathrm{C}_{1} \mathrm{C}_{3} \mathrm{O}_{1}\right)$.

To further evaluate the predictions of model 16, we simulated the decay phase of the macroscopic current after a $100 \mathrm{~ms}$ pulse of saturating agonists and compared it with experimentally measured decays. This decay depends on desensitization and ligand dissociation as well as the gating conformational changes (Lester and Jahr, 1992); hence, model 16 alone is insufficient. Using values from previous studies (Benveniste et al., 1990; Lester and Jahr, 1992; Popescu and Auerbach, 2003), we modified model 16 by adding binding and desensitizing steps.

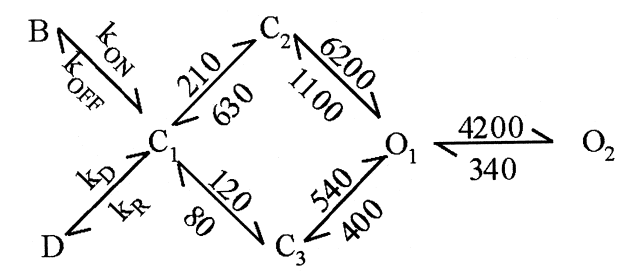

Model 16a

The rate constants are in $1 /$ seconds. B represents all less than fully liganded NMDARs, and D represents fully liganded, desensitized NMDARs. The agonist association and dissociation rate constants are $k_{\mathrm{ON}}$ and $k_{\mathrm{OFF}}$, and the entry and recovery rate constants for desensitization are $k_{\mathrm{D}}$ and $k_{\mathrm{R}}$. The dissociation rate constant $k_{\mathrm{OFF}}$ was chosen as $11.4 \mathrm{~s}^{-1}\left(2 \times k_{\mathrm{OFF} \_\mathrm{Glu}}+2 \times k_{\mathrm{OFF} \_\mathrm{Gly}}\right)$ (Benveniste et al., 1990; Lester and Jahr, 1992), and the desensitization/recovery rate constants were set at $4 / 1 \mathrm{~s}^{-1}$ (Popescu and Auerbach, 2003). The remaining rate constants were globally fitted using intervals within clusters from four outside-out patches. The desensitized state was connected, in turn, to each state of the scheme, and the best prediction of the decay phase of the NMDAR macroscopic current was obtained when D was connected to $\mathrm{C}_{1}$ (Fig. 5 c). The decay of NMDAR macroscopic current evoked by $100 \mathrm{~ms}$ pulse of $1 \mathrm{~mm}$ glutamate and $100 \mu \mathrm{M}$ glycine was fitted by the sum of two exponentials, with time constants (relative amplitudes) of $92.4 \pm 0.27 \mathrm{~ms}(85 \pm 0.3 \%)$ and $702.2 \pm$ $46.8 \mathrm{~ms}(15 \pm 0.2 \%)$, respectively $(n=3)$. The corresponding values for the best match with $\mathrm{D}$ connected to $\mathrm{C}_{1}$ were $88 \mathrm{~ms}$ $(83 \%)$ and $899 \mathrm{~ms}(17 \%)$. The next best match was D connected to $\mathrm{C}_{3}$, with values of $87 \mathrm{~ms}(65 \%)$ and $960 \mathrm{~ms}(35 \%)$. The predictions with $\mathrm{D}$ connected to states $\mathrm{C}_{2}, \mathrm{O}_{1}$, and $\mathrm{O}_{2}$ were significantly worse (only a single exponential). This result suggests that NMDAR desensitization occurs mainly from $\mathrm{C}_{1}$. Thus, according to model 16, there are three possible fully liganded pathways out of the first nonconducting state: two that lead to opening (after passing through a preopening closed state) and one that leads to desensitization.

We also simulated the response of NMDARs to short pulses of saturating glutamate (1-10 ms) using model 16 . The model predicts that the time it takes for the NMDAR current to reach its peak is independent of the length of pulse $(>1 \mathrm{~ms})$. As shown in Figure $5 c$, after the binding of agonists, the time to peak was 12.6 ms for currents evoked by 1,2, 4, and $8 \mathrm{~ms}$ pulses of saturating agonists. This conclusion is in accord with the observation that a pulse of saturating glutamate as short as $0.8 \mathrm{~ms}$ produces the maximal response of NMDARs (Lester and Jahr, 1992).

Model 16 shares a structure with the kinetic scheme proposed by Banke and Traynelis for the gating of NMDAR (Banke and Traynelis, 2003). We modified their scheme by adding a second, coupled open state. 


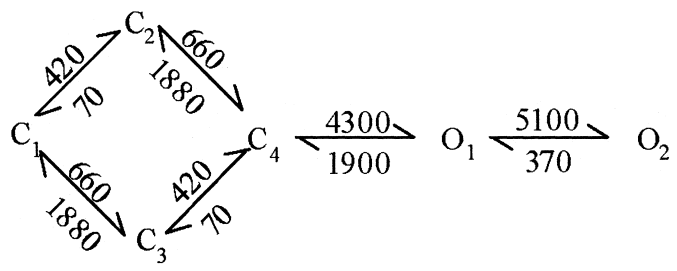

Model 17

Although model 17 contains four rather than three closed states, the pairs of rate constants on parallel sides of the cycle are constrained to be equal, yielding only eight free parameters. The optimal rate constants are shown in model 17.

In four patches, the average rate constants for the $\mathrm{C}_{1} \rightleftarrows \mathrm{C}_{2}$ and $\mathrm{C}_{2} \rightleftarrows \mathrm{C}_{4}$ steps in model 17 were essentially the same as the $\mathrm{C}_{1} \rightleftarrows \mathrm{C}_{2}$ and $\mathrm{C}_{2} \rightleftarrows \mathrm{C}_{3}$ rate constants obtained by using the linear, CCCOO scheme (model 11). Our estimate of the $\mathrm{C}_{2} \rightleftarrows \mathrm{C}_{4}$ equilibrium constant (0.35) is $>50$ times smaller than that reported for NR1/ NR2A NMDARs expressed in HEK cells at pH 7.3 (3140/174 $\mathrm{s}^{-1}=18$ ) (Erreger et al., 2005). Such discrepancy cannot be completely accounted for by the different $\mathrm{pHs}$ (8.0 vs 7.3) (Banke et al., 2005) and is perhaps the result of different expression systems.

\section{Discussion}

Our objective was to identify kinetic schemes that describe the gating reaction of fully liganded, recombinant NR1a/NR2A NMDARs. In general terms, the statistics-based modeling studies indicate that such a reaction mechanism must (1) contain at least three nonconducting and two conducting states, (2) have only one entry/exit gateway between the nonconducting and conducting aggregates, (3) have coupled conducting states, and (4) have at least two preopening, nonconducting states. We examined the ability of 17 different kinetic schemes to describe both steadystate single-channel currents and macroscopic currents elicited by a jump in agonist concentration. Only two noncyclic models were able to account for these channel behaviors, and these could be combined into a single cyclic scheme.

One of the two noncyclic schemes was a simple linear CCCOO mechanism that had been used previously to predict the NMDAR response to trains of synaptic impulses (Popescu and Auerbach, 2003; Popescu et al., 2004):

$$
\mathrm{C}_{1} \underset{-\frac{440}{75}}{\longrightarrow} \mathrm{C}_{2} \frac{640}{2000} \longrightarrow \mathrm{C}_{3} \frac{4800}{1600} \mathrm{O}_{1} \frac{4500}{350} \mathrm{O}_{2}
$$

Model 11a

The rate constants are in $1 /$ seconds. This reaction mechanism strikes us as being simpler than the alternative, branched model 8 , because with it NMDAR gating can be viewed as a straightforward, if sluggish, closed $\rightleftarrows$ open isomerization $\left(\mathrm{C}_{1} \ldots \rightleftarrows . . \mathrm{O}_{2}\right)$. NMDARs are large proteins in which the binding-site domains and the channel domain are separated by $>40 \AA$ (Miyazawa et al., 1999; Furukawa and Gouaux, 2003). Therefore, it is likely that intermediate conformations exist between the stable $\mathrm{C}_{1}$ and $\mathrm{O}_{2}$ end states of the reaction. In the linear scheme, some of the intermediate structures that populate the "transition state" of the overall reaction $\left(\mathrm{C}_{2}, \mathrm{C}_{3}\right.$, and $\left.\mathrm{O}_{1}\right)$ are sufficiently long-lived to be detected in patch-clamp recordings. In neuromuscular acetylcholine receptor channels, these intermediate conformations are, in general, too brief to be detected as discrete events and can be inferred only by using rate-equilibrium-free-energy relationship analysis (Auerbach, 2005). A preopening closed-intermediate state has also been observed directly in the glycine receptor channel (Burzomato et al., 2004). With the linear scheme, NMDARs can be thought of as closed-to-open gating devices that have long-lived intermediate states. Accordingly, the effective overall gating rates, which reflect the mean first-passage times between the stable end states of the reaction $\mathrm{C}_{1}$ and $\mathrm{O}_{2}$, are $5.64 \mathrm{~ms}$ (forward) and $228 \mathrm{~ms}$ (backward). The inverses of these values, 177 $\mathrm{s}^{-1}$ and $4.4 \mathrm{~s}^{-1}$, are the appropriate quantities for comparison with studies that model gating as a single, $\mathrm{C} \rightleftarrows \mathrm{O}$ step (Erreger et al., 2004).

The lifetimes of the intermediate preopening states $\mathrm{C}_{1}$ and $\mathrm{C}_{2}$ are remarkably long (1-2 ms). These states acts as "way stations" that serve to slow both the rise and the decay of the synaptic current. Moreover, the lifetime of $\mathrm{C}_{1}$ is longer than that of a transmitter pulse and allows a single NMDAR to integrate information from multiple synaptic impulses (Popescu and Auerbach, 2003). We speculate that natural selection has operated on the stability of states $C_{1}$ and $C_{2}$ to enforce these critical pauses in the NMDAR gating reaction.

With two notable exceptions, the conductance and kinetic parameters for NR1a/NR2A NMDARs expressed in oocytes (outside-out patches) or in HEK cells (M-mode; cell-attached patches) (Popescu and Auerbach, 2003), under otherwiseidentical experimental conditions, are similar. One important difference is that we observed only one pattern of activity rather than three, as in HEK cells. The reason for this difference is not clear. One possibility is that GFP, coexpressed with the NMDAR subunits only in HEK cells, is responsible. However, the difference might arise from the expression systems themselves. It is known that different expression systems can differ in their translational efficiency, posttranslational modifications such as glycosylation and phosphorylation, subunit or associated-protein assembly, or even lipid environment. For example, it has been reported that there are substantial differences in the kinetics of ClC-2 channels expressed in oocytes versus HEK cells (Thiemann et al., 1992; Jordt and Jentsch, 1997; Park et al., 1998), which can partly be explained by difference in phosphorylation. In another study, it was found that the glycosylation patterns are different between native nicotinic receptors of Torpedo californica and those expressed in oocytes (Buller and White, 1990). The multiple activation patterns of NMDARs expressed in HEK cells could be a potential target for modulation, because the open probability of NMDARs in each mode is quite different. If mode switching is a mechanism that can be used to tune the activity of NMDARs in the CNS, it will be interesting to determine whether or not this behavior is a function of the expression system and, if so, which processes govern its occurrence.

The second significant difference between the oocyte and HEK cell kinetic parameters is the kinetics of the $\mathrm{C}_{1} \rightleftarrows \mathrm{C}_{2}$ transition. (All other rate constants are within a factor of 2.) In the oocyte experiments, the rate constants for this step were 440/75 $\mathrm{s}^{-1}$ (forward/backward; yielding an equilibrium constant of 5.3), whereas in the HEK experiments, these were 150/173 $\mathrm{s}^{-1}$ (yielding an equilibrium constant of 0.9 , independent of mode). This difference predicts a faster rise and slower decay of the synaptic impulse response using the oocyte values and is thus worth noting, although we have no information regarding the mechanistic basis for this discrepancy.

The cyclic scheme we explored (model 16) has the same general structure as the gating scheme proposed by Banke and Traynelis (2003) for the activation of recombinant NR1/NR2B NMDARs (expressed in HEK), although the methods used to 
deduce these two models were quite different. The BT model was based on the observation that partial agonists for glutamate and glycine binding sites alter different components of the interval duration distribution of intracluster closed events, which led to the speculation that, during the gating reaction, the NR2 and NR1 subunits change their conformations independently. In our experiments, model 16 was investigated simply because it incorporated both of the two best noncyclic schemes, and was not based on any assumptions regarding NMDAR structure or gating mechanism. Nevertheless, the similarity of these models, obtained by using completely different approaches, suggests that a dual pathway mechanism for gating is possible. In model $16, \mathrm{C}_{1}$, $\mathrm{C}_{2}$, and $\mathrm{C}_{3}$ act as way stations. The lifetime of $\mathrm{C}_{2}(0.15 \mathrm{~ms})$ is much shorter than that of $\mathrm{C}_{3}(1.6 \mathrm{~ms})$, but $\mathrm{C}_{2}$ is visited approximately twice as frequently as $\mathrm{C}_{3}$ within the activation sequence (both forward and backward).

At this stage, we cannot unambiguously associate the kinetic events with particular changes in the conformations of structural domains. Given that glutamate and glycine analogs specifically alter the $\mathrm{C}_{1} \rightleftarrows \mathrm{C}_{2}$ and $\mathrm{C}_{2} \rightleftarrows \mathrm{C}_{3}$ transitions, it is likely that these events reflect motions of the S1-S2 domains of the NR2 and NR1 subunits (Banke and Traynelis, 2003; Jin et al., 2003). It is uncertain whether or not these preopening isomerizations pertain to conformational changes of entire subunits, because they may also reflect the motions of other domains of the NMDAR ("blocks") that may or may not be subunit delimited (Chakrapani et al., 2004). We must wait for additional kinetic and other functional analyses of NMDARs that have been perturbed by ligands and mutations before this distinction can be made with confidence.

Although the models we selected adequately account for the data, there are several noteworthy limitations to our study. The currents were not recorded under physiological conditions and therefore the models do not incorporate divalent cation inhibition (Mayer et al., 1984; Nowak et al., 1984) or subconductance levels (Stern et al., 1992, 1994). Ligand binding and desensitization steps were not included in the modeling, although the incorporation of published values for these parameters made accurate predictions with regard to experimental current-decay time courses. Nonetheless, we hope that the information provided by this study will facilitate our future understanding of how NMDARs are used for signal transduction at synapses and how NMDARs operate as protein nanomachines.

\section{References}

Auerbach A (2005) Gating of acetylcholine receptor channels: brownian motion across a broad transition state. Proc Natl Acad Sci USA 102:1408-1412.

Banke TG, Traynelis SF (2003) Activation of NR1/NR2B NMDA receptors. Nat Neurosci 6:144-152.

Banke TG, Dravid SM, Traynelis SF (2005) Protons trap NR1/NR2B NMDA receptors in a nonconducting state. J Neurosci 25:42-51.

Behe P, Stern P, Wyllie DJ, Nassar M, Schoepfer R, Colquhoun D (1995) Determination of NMDA NR1 subunit copy number in recombinant NMDA receptors. Proc R Soc Lond B Biol Sci 262:205-213.

Benveniste M, Mayer ML (1991a) Kinetic analysis of antagonist action at $\mathrm{N}$-methyl-D-aspartic acid receptors. Two binding sites each for glutamate and glycine. Biophys J 59:560-573.

Benveniste M, Mayer ML (1991b) Structure-activity analysis of binding kinetics for NMDA receptor competitive antagonists: the influence of conformational restriction. Br J Pharmacol 104:207-221.

Benveniste M, Clements J, Vyklicky Jr L, Mayer ML (1990) A kinetic analysis of the modulation of $N$-methyl-D-aspartic acid receptors by glycine in mouse cultured hippocampal neurones. J Physiol (Lond) 428:333-357.

Buller AL, White MM (1990) Altered patterns of N-linked glycosylation of the Torpedo acetylcholine receptor expressed in Xenopus oocytes. J Membr Biol 115:179-189.
Burzomato V, Beato M, Groot-Kormelink PJ, Colquhoun D, Sivilotti LG (2004) Single-channel behavior of heteromeric $\alpha 1 \beta$ glycine receptors: an attempt to detect a conformational change before the channel opens. J Neurosci 24:10924-10940.

Chakrapani S, Bailey TD, Auerbach A (2004) Gating dynamics of the acetylcholine receptor extracellular domain. J Gen Physiol 123:341-356.

Clapham DE, Neher E (1984) Substance P reduces acetylcholine-induced currents in isolated bovine chromaffin cells. J Physiol (Lond) 347:255-277.

Clements JD, Lester RA, Tong G, Jahr CE, Westbrook GL (1992) The time course of glutamate in the synaptic cleft. Science 258:1498-1501.

Colquhoun D, Hawkes AG (1981) On the stochastic properties of single ion channels. Proc R Soc Lond B Biol Sci 211:205-235.

Colquhoun D, Hawkes AG (1982) On the stochastic properties of bursts of single ion channel openings and of clusters of bursts. Philos Trans R Soc Lond B Biol Sci 300:1-59.

Colquhoun D, Hawkes AG (1987) A note on correlations in single ion channel records. Proc R Soc Lond B Biol Sci 230:15-52.

Colquhoun D, Hawkes AG (1995a) The principles of the stochastic interpretation of ion-channel mechanisms. In: Single-channel recording (Sakman B, Neher E, eds), pp 397-482. New York: Plenum.

Colquhoun D, Hawkes AG (1995b) A Q-matrix cookbook: how to write only one program to calculate the single-channel and macroscopic predictions for any kinetic mechanism. In: Single-channel recording (Sakman B, Neher E, eds), pp 589-633. New York: Plenum.

Colquhoun D, Sakmann B (1985) Fast events in single-channel currents activated by acetylcholine and its analogues at the frog muscle end-plate. J Physiol (Lond) 369:501-557.

Dingledine R, Borges K, Bowie D, Traynelis SF (1999) The glutamate receptor ion channels. Pharmacol Rev 51:7-61.

Erreger K, Chen PE, Wyllie DJ, Traynelis SF (2004) Glutamate receptor gating. Crit Rev Neurobiol 16:187-224.

Erreger K, Dravid SM, Banke TG, Wyllie DJ, Traynelis SF (2005) Subunitspecific gating controls rat NR1/NR2A and NR1/NR2B NMDA channel kinetics and synaptic signalling profiles. J Physiol (Lond) 563:345-358.

Fredkin DR, Montal M, Rice JA (1985) Identification of aggregated Markovian models: application to the nicotinic acetylcholine receptor. (LeCam LM, Olshen RA, eds), pp 269-289. Belmont, CA: Wadsworth.

Furukawa H, Gouaux E (2003) Mechanisms of activation, inhibition and specificity: crystal structures of the NMDA receptor NR1 ligand-binding core. EMBO J 22:2873-2885.

Gibb AJ, Colquhoun D (1991) Glutamate activation of a single NMDA receptor-channel produces a cluster of channel openings. Proc R Soc Lond B Biol Sci 243:39-45.

Gibb AJ, Colquhoun D (1992) Activation of N-methyl-D-aspartate receptors by L-glutamate in cells dissociated from adult rat hippocampus. J Physiol (Lond) 456:143-179.

Gil Z, Magleby KL, Silberberg SD (2001) Two-dimensional kinetic analysis suggests nonsequential gating of mechanosensitive channels in Xenopus oocytes. Biophys J 81:2082-2099.

Hamill OP, Marty A, Neher E, Sakmann B, Sigworth FJ (1981) Improved patch-clamp techniques for high-resolution current recording from cells and cell-free membrane patches. Pflugers Arch 391:85-100.

Hollmann M, Heinemann S (1994) Cloned glutamate receptors. Annu Rev Neurosci 17:31-108.

Jin R, Banke TG, Mayer ML, Traynelis SF, Gouaux E (2003) Structural basis for partial agonist action at ionotropic glutamate receptors. Nat Neurosci 6:803-810.

Jonas P (1995) Fast application of agonists to isolated membrane patches. In: Single-channel recording (Sakman B, Neher E, eds), pp 231-243. New York: Plenum.

Jordt SE, Jentsch TJ (1997) Molecular dissection of gating in the ClC-2 chloride channel. EMBO J 16:1582-1592.

Kleckner NW, Dingledine R (1988) Requirement for glycine in activation of NMDA-receptors expressed in Xenopus oocytes. Science 241:835-837.

Labarca P, Rice JA, Fredkin DR, Montal M (1985) Kinetic analysis of channel gating. Application to the cholinergic receptor channel and the chloride channel from Torpedo californica. Biophys J 47:469-478.

Lester RA, Jahr CE (1992) NMDA channel behavior depends on agonist affinity. J Neurosci 12:635-643.

Magleby KL, Pallotta BS (1983) Burst kinetics of single calcium-activated potassium channels in cultured rat muscle. J Physiol (Lond) 344:605-623. 
Magleby KL, Song L (1992) Dependency plots suggest the kinetic structure of ion channels. Proc R Soc Lond B Biol Sci 249:133-142.

Mayer ML, Westbrook GL, Guthrie PB (1984) Voltage-dependent block by $\mathrm{Mg}^{2+}$ of NMDA responses in spinal cord neurones. Nature 309:261-263.

Miyazawa A, Fujiyoshi Y, Stowell M, Unwin N (1999) Nicotinic acetylcholine receptor at $4.6 \AA$ resolution: transverse tunnels in the channel wall. J Mol Biol 288:765-786.

Nowak L, Bregestovski P, Ascher P, Herbet A, Prochiantz A (1984) Magnesium gates glutamate-activated channels in mouse central neurones. $\mathrm{Na}$ ture 307:462-465.

Park K, Arreola J, Begenisich T, Melvin JE (1998) Comparison of voltageactivated Cl-channels in rat parotid acinar cells with ClC-2 in a mammalian expression system. J Membr Biol 163:87-95.

Patlak JB (1988) Sodium channel subconductance levels measured with a new variance-mean analysis. J Gen Physiol 92:413-430.

Popescu G, Auerbach A (2003) Modal gating of NMDA receptors and the shape of their synaptic response. Nat Neurosci 6:476-483.

Popescu G, Auerbach A (2004) The NMDA receptor gating machine: lessons from single channels. Neuroscientist 10:192-198.

Popescu G, Robert A, Howe JR, Auerbach A (2004) Reaction mechanism determines NMDA receptor response to repetitive stimulation. Nature 430:790-793.

Premkumar LS, Auerbach A (1996) Identification of a high affinity divalent cation binding site near the entrance of the NMDA receptor channel. Neuron 16:869-880.

Qin F (2004) Restoration of single-channel currents using the segmental k-means method based on hidden Markov modeling. Biophys J 86:1488-1501.

Qin F, Auerbach A, Sachs F (1996) Estimating single-channel kinetic parameters from idealized patch-clamp data containing missed events. Biophys J 70:264-280.

Rosenmund C, Stern-Bach Y, Stevens CF (1998) The tetrameric structure of a glutamate receptor channel. Science 280:1596-1599.

Sakmann B, Patlak J, Neher E (1980) Single acetylcholine-activated chan- nels show burst-kinetics in presence of desensitizing concentrations of agonist. Nature 286:71-73.

Sigworth FJ, Sine SM (1987) Data transformations for improved display and fitting of single-channel dwell time histograms. Biophys J 52:1047-1054.

Song L, Magleby KL (1994) Testing for microscopic reversibility in the gating of maxi $\mathrm{K}^{+}$channels using two-dimensional dwell-time distributions. Biophys J 67:91-104.

Stern P, Behe P, Schoepfer R, Colquhoun D (1992) Single-channel conductances of NMDA receptors expressed from cloned cDNAs: comparison with native receptors. Proc R Soc Lond B Biol Sci 250:271-277.

Stern P, Cik M, Colquhoun D, Stephenson FA (1994) Single channel properties of cloned NMDA receptors in a human cell line: comparison with results from Xenopus oocytes. J Physiol (Lond) 476:391-397.

Sun Y, Olson R, Horning M, Armstrong N, Mayer M, Gouaux E (2002) Mechanism of glutamate receptor desensitization. Nature 417:245-253.

Thiemann A, Grunder S, Pusch M, Jentsch TJ (1992) A chloride channel widely expressed in epithelial and non-epithelial cells. Nature 356:57-60.

Vicini S, Wang JF, Li JH, Zhu WJ, Wang YH, Luo JH, Wolfe BB, Grayson DR (1998) Functional and pharmacological differences between recombinant $N$-methyl-D-aspartate receptors. J Neurophysiol 79:555-566.

Villarroel A, Regalado MP, Lerma J (1998) Glycine-independent NMDA receptor desensitization: localization of structural determinants. Neuron 20:329-339.

Wollmuth LP, Sakmann B (1998) Different mechanisms of $\mathrm{Ca}^{2+}$ transport in NMDA and $\mathrm{Ca}^{2+}$-permeable AMPA glutamate receptor channels. J Gen Physiol 112:623-636.

Wollmuth LP, Kuner T, Sakmann B (1998) Intracellular $\mathrm{Mg}^{2+}$ interacts with structural determinants of the narrow constriction contributed by the NR1-subunit in the NMDA receptor channel. J Physiol (Lond) 506:33-52.

Wyllie DJ, Behe P, Colquhoun D (1998) Single-channel activations and concentration jumps: comparison of recombinant NR1a/NR2A and NR1a/NR2D NMDA receptors. J Physiol (Lond) 510:1-18. 